Epochen der deutschen Literatur

Materialienband

Realismus und Gründerzeit

Manifeste und Dokumente zur deutschen

Literatur 1848-1880

Band 2 


\section{Realismus und Gründerzeit}

\section{Manifeste und Dokumente zur deutschen Literatur 1848 - 1880}

Mit einer Einführung in den Problemkreis und einer Quellenbibliographie herausgegeben von

Max Bucher, Werner Hahl, Georg Jäger

und Reinhard Wittmann

Band 2

Manifeste und Dokumente 
ISBN 978-3-476-00267-9

ISBN 978-3-476-03017-7 (eBook)

DOI 10.1007/978-3-476-03017-7

(C) Springer-Verlag GmbH Deutschland 1975

Ursprünglich erschienen bei J. B. Metzlersche Verlagsbuchhandlung und Carl Ernst Poeschel Verlag GmbH in Stuttgart 1975 
Das Inhaltsverzeichnis enthält zur leichteren Orientierung verkürzte und vereinfachte Titelangaben. Die genauen Nachweise stehen über den Dokumenten. Sie geben Auskunft über die Kürzungen und die abgedruckten Ausschnitte, über Anonyme und Pseudonyme. Der Zeitschriftenfundort wird hier nur hinzugefügt, wo die Verfasser nicht ermittelt werden konnten. Die Jahreszahl bezeichnet die (Erst-)Veröffentlichung, die als Druckvorlage dient. Die maßgeblichen Neudrucke finden sich in den Angaben über den Dokumenten.

\section{Der Realismusbegriff in der Kunst \\ Religionskritik, Asthetik und Geschichte}

1 Friedrich Theodor Vischer: Rez. Friedrich Overbeck, Der Triumph der Religion in den Künsten. 1841 . . . . . . . . . . . . . . . . . . . 2

2 Anton Springer: Kritische Gedanken über die Münchner Kunst. 1845 . . . 5

3 David Friedrich Strauß: Aesthetische Grillen. 1848 . . . . . . . . . . 7

4 Hermann Hettner: Drangsale und Hoffnungen der modernen Plastik. 1846 . . 9

\section{Die Diskussion um die Historienmalerei}

5 Friedrich Theodor Vischer: Die Aquarell-Copien von Ramboux in der Gallerie zu Düsseldorf. Einleitende Betrachtungen über den Zustand der jetzigen Malerei. 1842 . . . . . . . . . . . . . . . . . . . . . . . 19

6 Anton Springer: Die geschichtliche Malerei in der Gegenwart. 1846 . . . . 21 beiden Bilder von Gallait und de Biefve. 1843 . . . . . . . . . . . 26

7 Franz Kugler: Sendschreiben an Herrn Dr. Ernst Förster in München über die 8 Anton Teichlein: Louis Gallait und Die Malerei in Deutschland. 1853 . . . 30

9 Max Schasler: Ueber Idealismus und Realismus in der Historienmalerei. $1858 \quad 34$

\section{Die Plastik als Vorbild der Dichtung}

10 Anton Gubitz: Kunst und Künstler in Berlin. 1851 . . . . . . . . . . 39

11 Theodor Fontane: Unsere lyrische und epische Poesie seit 1848. 1853 . . . . 40

\section{Idealrealismus und Panentheismus}

12 Aus Ernst Rietschels Notizbüchern. 1863 . . . . . . . . . . . . . 42 
13 Moriz Carriere: Die Aufgabe der Kunst in der Gegenwart. 1857 . . . . 43

14 Melchior Meyr: Die deutsche Kunst in der münchener Ausstellung. 1859 . . 46

15 Adalbert Stifter: Ausstellung des oberösterreichischen Kunstvereines. 1867 . 48

\author{
Der Realismus in der Literatur \\ (Vorbereitung, Programm, Kritik)
}

\title{
Revolutionsprogramme
}

16 Heinrich Landesmann (Ps. Hieronymus Lorm): Poesie und Politik. 1848 - . 52

17 Ferdinand Kürnberger: Gedanken über die Lyrik der Zukunft. 1848 . . . . 54

18 Richard Wagner: Die Kunst und die Revolution. 1849 . . . . . . . . . 58

19 Hermann Hettner: Die Kunst und ihre Zukunft. 1850 . . . . . . . . . 61

Der programmatische Realismus der "Grenzboten « und verwandte realistische Positionen Volk und Nation als Basis des neuen Realismus

20 Hermann Hettner: Die romantische Schule in ihrem inneren Zusammenhange mit Goethe und Schiller. 1850 . . . . . . . . . . . . . . . . . 64

21 Deutsche Dorfgeschichten. In: Jahrbücher für Wissenschaft und Kunst, 1854 . 67

22 Julian Schmidt: Rez. Leo Cholevius, Geschichte der deutschen Poesie nach ihren antiken Elementen. 1856 . . . . . . . . . . . . . . . . 69

Realismus und Bürgertum: Das sittliche Prinzip in der Darstellung der Arbeitswelt

23 Gustav Freytag: Deutsche Romane. 1853 . . . . . . . . . . . . . 71

24 Gustav Freytag: Neue deutsche Romane. 1853 . . . . . . . . . . 72

25 Julian Schmidt: »Wilhelm Meister« im Verhältniß zu unsrer Zeit. 1855 . . . 73

Literatur und Revolution: Die Abrechnung mit der Literatur der Restaurationszeit

26 Friedrich Theodor Vischer: Noch ein Wort darüber, warum ich von der jetzigen Poesie nichts halte. 1844 . . . . . . . . . . . . . . . . 75

27 Julian Schmidt: Die Märzpoeten. 1850 . . . . . . . . . . . . . 78

28 Julian Schmidt: Die Reaction in der deutschen Poesie. 1851 . . . . . . . 83

Die Ausbildung des Realismusbegriffs

29 Julian Schmidt: Georg Büchner. 1851 . . . . . . . . . . . . . 87

30 Julian Schmidt: Einige Uebelstände in unsrem Theaterwesen. 1852 . . . 88

31 Julian Schmidt: Der neueste englische Roman und das Princip des Realismus. 1856 . . . . . . . . . . . . . . . . . . . . . . . . . 90

32 Julian Schmidt: Schiller und der Idealismus. 1858 . . . . . . . . . . 94 
33 Julian Schmidt: Neue Romane. 1860 . . . . . . . . . . . . . . 96

34 Theodor Fontane: Unsere lyrische und epische Poesie seit 1848. 1853 . . . . 98

35 Otto Ludwig: Shakespeare-Studien. (Aus dem Nachlaß.) . . . . . . . . 101

36 Berthold Auerbach: Rez. Gottfried Keller, Die Leute von Seldwyla. 1856 . . 104

37 Karl Frenzel: Das >Moderne in der Kunst. 1868 . . . . . . . . . . . 108

Forderungen nach einer Synthese von Idealismus und Realismus: Der Realidealismus

38 Karl Gutzkow: Realismus und Idealismus. 1857 . . . . . . . . . . . 111

39 Rudolph Gottschall: Poetik. 1858 . . . . . . . . . . . . . . . 112

40 Adolf Helfferich: Das Stilgesetz in den bildenden Künsten. 1863 . . . . . 115

41 Heinrich Emil Homberger: Der realistische Roman. 1870 . . . . . . . . 117

Der religionsphilosophische Streit um den Realismus

Der Pantheismus

42 Friedrich Theodor Vischer: Akademische Rede zum Antritte des Ordinariats. 1845

43 Theodor Mundt: Aesthetik. 1845 . . . . . . . . . . . . . . . 124

Die panentheistische Ästhetik als Theologie der Verklärung

44 Melchior Meyr: Gedichte 1857 . . . . . . . . . . . . . . . . 127

45 Moriz Carriere: Aesthetik. 1859 . . . . . . . . . . . . . . . . 129

46 Melchior Meyr: Emilie. Drei Gespräche über Wahrheit, Güte und Schönheit.

1863 . . . . . . . . . . . . . . . . . . . . . . . . . 132

47 Ludwig Eckard: Vorschule der Aesthetik. 1864 . . . . . . . . . . . 135

Idealismus und Aristokratismus

48 Hermann Grimm: Rafael und Michelangelo. 1859 . . . . . . . . . . 139

49 Wilhelm Scherer: Bürgerthum und Realismus. 1870 . . . . . . . . . 141

50 Emerich du Mont: Der Fortschritt im Lichte der Lehren Schopenhauers und Darwins. 1876. . . . . . . . . . . . . . . . . . . . . . 143

\section{Die Dorfgeschichte}

\section{Dorfgeschichte und Nationalgedanke im Vormärz}

51 Berthold Auerbach: An J. E. Braun vom Verfasser der "Schwarzwälder Dorfgeschichten ${ }^{2} 1843$. . . . . . . . . . . . . . . . . . . . 148

52 Karl Hagen: Berthold Auerbachs "Schwarzwälder Dorfgeschichten«. 1844 . . 152

53 Ferdinand Gustav Kühne: Vorwort zur Zeitschrift »Europa«. 1846 . . . . 154

54 St. René Taillandier: Úber Roman und Kritik in Deutschland. 1846 . . . . 155 
Der Liberalismus und die Ästhetik des Volkstümlichen

55 Berthold Auerbach: Schrift und Volk. 1846 . . . . . . . . . . . . 158

56 Ferdinand Kürnberger: Ein Votum über die Literatur der Dorfgeschichten. 1848163

57 Carl Dolde: Gegen »ein Votum über die Literatur der Dorfgeschichten von Ferdinand Kürnberger « 1848 . . . . . . . . . . . . . . . . . . 166

58 Ferdinand Kürnberger: Leopold Kompert. 1848 . . . . . . . . . . . 167

59 Gottfried Keller: Jeremias Gotthelf. 1849 . . . . . . . . . . . . . 168

\section{Gesellschaftlicher Konservatismus im Nachmärz}

60 Julian Schmidt: Geschichte der deutschen Literatur im neunzehnten Jahrhundert. 1855. (Zu Auerbach und Gotthelf.) . . . . . . . . . . . . . 176

61 Deutsche Dorfgeschichten. In: Jahrbücher für Wissenschaft und Kunst, 1854 . 178

62 Die Dorfgeschichte. In: Weimarer Sonntagsblatt, 1857 . . . . . . . . 182

63 Gustav Hauff: Uber Dorfgeschichten. 1859 . . . . . . . . . . . . 185

\section{Kritik der verklärten Primitivität}

64 Friedrich Theodor Vischer: Ästhetik. Die Dichtkunst. 1857. (Zu Auerbachs "Dorfgeschichten" und Goethes "Hermann und Dorothea «.) . . . . . . 189

65 Julian Schmidt: Otto Ludwig. 1857 . . . . . . . . . . . . . . . 190

66 Heinrich Treitschke: Otto Ludwig. 1859 . . . . . . . . . . . . . 193

67 Gustav Freytag: Deutsche Dorfgeschichten. 1862 . . . . . . . . . 196

68 Karl Gutzkow: Die realistischen Erzähler. 1857 . . . . . . . . . . . 197

69 Rudolph Gottschall: Rez. Berthold Auerbach, Barfüßele. 1857 . . . . . . 200

\section{Klassische und romantische Interpretationsschemata}

70 Rez. Berthold Auerbach, Barfüßele. In: Literatur-Blatt des Deutschen Kunstblattes, 1857 . . . . . . . . . . . . . . . . . . . . . . 204

71 J. F. Faber: Riehls"Geschichten aus alter Zeit«. 1863 . . . . . . . . . 207

72 Karl Stieler: Volksausgabe von Auerbachs Dorfgeschichten. 1871 . . . . . 211

\section{Roman und Novelle}

Roman und Gesellschaft

Epik in der verwalteten Welt

73 Friedrich Theodor Vischer: Ästhetik. Die Dichtkunst. 1857. (Zum Roman) . 216

\section{Goethes Gesellschattsroman im Nachmärz}

74 Hermann Hettner: Goethe und der Socialismus. 1852 . . . . . . . . . 221

75 Julian Schmidt: »Wilhelm Meister« im Verhältniß zu unsrer Zeit. 1855 . . . 226 
Roman und staatsbürgerliches Bewußtsein

76 Jeremias Gotthelf: Erlebnisse eines Schuldenbauers, Vorwort. 1853 . . . . 232

77 Adolf Stahr: Rez. Wilibald Alexis, Ruhe ist die erste Bürgerpflicht. 1852 . . 234

78 Karl Hillebrand: Vom alten und neuen Roman. 1884 . . . . . . . . 237

Das Problem des gesellschattlichen Pluralismus

79 Adolf Rutenberg: Der deutsche Roman der Gegenwart. 1873 . . . . . . 243

80 Friedrich Spielhagen: Der Ich-Roman. 1881-82 . . . . . . . . . . . 249

Roman und soziale Integration

81 Otto Ludwig: Volksroman - Volkslitteratur. (Aus dem Nachlaß.) . . . . . 258

82 Otto Ludwig: "Harte Zeiten« von Dickens. (Aus dem Nachlaß.) . . . . . 262

83 Ludwig Walesrode: Rez. Berthold Auerbach, Auf der Höhe. 1865 . . . 263

84 Erwin Schlieben: Zur Theorie des Romans. 1876 . . . . . . . . . . 269

\section{Roman und Geschichte}

\section{Der nationalhistorische Roman}

85 Julian Schmidt: Der vaterländische Roman. 1852 . . . . . . . . . . 278

86 Wilibald Alexis: Isegrimm, Vorrede und Schlußwort. 1854 . . . . . . . 282

87 Gustav Freytag: Rez. Wilibald Alexis, Isegrimm. 1854 . . . . . . . . 285

88 Theodor Fontane: Wilibald Alexis. 1872 . . . . . . . . . . . . . 288

\section{Geschichtlicher Stoff und modernes Bewußtsein}

89 Julian Schmidt: Rez. Charles Kingsley, Westward ho! 1856 . . . . . . . 290

90 Otto Ludwig: Walter Scotts Einheitlichkeit. Seine Durchschnittshelden. (Aus dem Nachlaß.) . . . . . . . . . . . . . . . . . . . . . 292

91 Joseph Victor Scheffel: Ekkehard, Vorwort. 1855 . . . . . . . . . . 294

\section{Populäre Romangattungen}

Der biographische Roman

92 Adolf Stern: Der bbiographischer Roman. 1863 . . . . . . . . . . . 296

93 Karl Frenzel: Der historische Roman. 1866 . . . . . . . . . . . . 299

Der Kriminalroman

94 Adolf Stern: Der Kriminalroman und die Kriminalnovelle. 1864 . . . . . 301

95 Adolf Rutenberg: Der Criminalroman und das Zeitalter des Modernen. 1874 . 304 
Kontroversen

Gutzkows allegorischer Zeitroman

96 Karl Gutzkow: Die Ritter vom Geiste, Vorwort. 1850 . . . . . . . . . 312

97 Julian Schmidt: Rez. Karl Gutzkow, Die Ritter vom Geiste, 2. Aufl. 1852 314

Der Roman und die Arbeit (G. Freytag)

98 Gustav Freytag: Soll und Haben, Motto und Widmung. 1855 . . . . . . 323

99 Karl Gutzkow: Rez. Gustav Freytag, Soll und Haben. 1855 . . . . . . . . 324

100 Theodor Fontane: Rez. Gustav Freytag, Soll und Haben. 1855 . . . . . 328

101 Robert Giseke: Rez. Gustav Freytag, Soll und Haben. 1855 . . . . . . . . 336

102 Hermann Marggraff: Rez. Gustav Freytag, Soll und Haben. 1855 . . . . . 340

103 Julian Schmidt: Geschichte der deutschen Literatur. 4. Aufl. 1858. (Zu Freytag, Soll und Haben.) . . . . . . . . . . . . . . . . . . . . . 343

104 Rudolph Gottschall: Rez. Gustav Freytag, Die verlorene Handschrift. 1865 . 346

105 Michael Bernays: Charakteristik von Gustav Freytags Roman "Die verlorene Handschrift«. 1865 . . . . . . . . . . . . . . . . . . . . 349

Le roman expérimental (Zola)

106 Ludwig Pfau: Emile Zola. 1880 . . . . . . . . . . . . . . . . 352

107 Michael Georg Conrad: Zola und Daudet. 1880 . . . . . . . . . . . 359

Die Novelle

Realistische Erneuerung

108 Friedrich Theodor Vischer: Ästhetik. Die Dichtkunst. 1857. (Zur Novelle.) . . 363

109 Hermann Hettner: Die romantische Schule in ihrem inneren Zusammenhange mit Goethe und Schiller. 1850 . . . . . . . . . . . . . . . . . 364

110 Friedrich Theodor Vischer: Gottfried Keller. 1874 . . . . . . . . . . 366

111 Theodor Storm: Eine zurückgezogene Vorrede aus dem Jahre 1881 . . . 368

Die Kontroverse Riehl - Heyse

112 Wilhelm Heinrich Riehl: Geschichten aus alter Zeit, Widmung. 1863 . . . . 369

113 Paul Heyse und Hermann Kurz: Deutscher Novellenschatz, Einleitung. 1871 . 370

114 Paul Heyse: An Frau Toutlemonde in Berlin. 1869 . . . . . . . . . . 372

115 Wilhelm Heinrich Riehl: Novelle und Sonate. 1885 . . . . . . . . . . 377

\section{Die Erzähltheorie}

Formen der Erzäblung

116 Otto Ludwig: Formen der Erzählung. (Aus dem Nachlaß.) . . . . . . . 379 
Das objektive Erzäblen

117 Julian Schmidt: Rez. Gottfried Keller, Der Grüne Heinrich. 1854 . . 381

118 Julian Schmidt: Rez. William M. Thackeray, The Newcomes. 1856 . • . 382

119 Friedrich Spielhagen: Der Held im Roman. 1874/1883 . . . . . . . . 384

\section{Verteidigung des Erzählers}

120 Berthold Auerbach: Goethe und die Erzählungskunst. 1861 . . . . . . . 388

121 Berthold Auerbach: Der Pfarrer von Wakefield. 1865 . . . . . . . . . 390

\section{Winke für literarische Handwerker}

122 Julius Duboc: Úber die Darstellungsweise im Roman. 1871 . . . . . . . 394

123 Gustav Freytag: Für junge Novellendichter. 1872 . . . . . . . . . . 397

\section{Versepik und Dramatik}

\section{Programme zu einem Gegenwartsepos}

124 Robert Prutz: Rez. Theodor Mundt, Thomas Müntzer. 1842 . . . . . . 402

125 Gegenwart und Zukunft des Epos in Deutschland. In: Literarische Zeitung, 1846 . . . . . . . . . . . . . . . . . . . . . . . 405

126 Max Waldau: Neuere epische Dichtung und Rudolph Gottschall. 1854 . . . 407

127 J. F. Faber: Das moderne Epos. 1855 . . . . . . . . . . . . . . 412

Die Epostheorie des Klassizismus und Spätrealismus

128 Rudolph Gottschall: Die deutsche Nationalliteratur in der ersten Hälfte des neunzehnten Jahrhunderts. 1855 . . . . . . . . . . . . . . . . 415

129 Rudolph Gottschall: Poetik. 1858 . . . . . . . . . . . . . . 416

130 Moriz Carriere: Die Poesie. 1884 . . . . . . . . . . . . . . 421

Staat, Gesellschaft und Drama nach 1848

131 Robert Prutz: Das Drama der Gegenwart. 1851 . . . . . . . . . . . 424

132 Ludwig Eckardt: Ueber das deutsche Drama der Neuzeit. 1858 . . . . . . 428

\section{Die Technik des Dramas}

133 Gustav Freytag: Die Technik des Dramas. 1849 . . . . . . . . . . . 432

\section{Die Kritik an Hebbel}

134 Heinrich Treitschke: Friedrich Hebbel. 1860 . 
135 Adolf Strodtmann: Friedrich Hebbel. 1862 . . . . . . . . . . 442

136 Robert Prutz: Friedrich Hebbel. 1864 . . . . . . . . . . . . . . 445

\section{Der Klassizismus im Drama}

137 Constantin Rössler: Gustav Freytag und die deutsche Dichtung der Gegenwart. 1860 . . . . . . . . . . . . . . . . . . . . . . .

138 Antike Dramenstoffe auf der deutschen Bühne. In: Literatur-Blatt des Deutschen Kunstblattes, 1858 . . . . . . . . . . . . . . . . . . 450

139 Rudolph Gottschall: Die französisirende Richtung der neuern deutschen Dramatik und Dramaturgie. 1857 . . . . . . . . . . . . . . . . . 454

140 Julian Schmidt: Antike Versuche auf dem modernen Theater. 1857 . . . 456

\section{Das bürgerliche Drama}

141 Emil Müller-Samswegen: Das bürgerliche Drama. 1858 . . . . . . . 460

142 Ferdinand Kürnberger: Rez. Friedrich Hebbel, Maria Magdalena. 1848 . . 465

143 Heinrich Treitschke: Otto Ludwig. 1859 . . . . . . . . . . . . . 468

\section{Das Geschichtsdrama}

144 Karl Gutzkow: Vorrede zu »Wullenweber«. 1848 . . . . . . . . . . 470

145 Rudolph Gottschall: Ueber die historische Tragödie. 1859 . . . . . . 471

146 Melchior Meyr: Die Gefahr und das Heil des deutschen Dramas. 1868 . . 475

\section{Die Meininger: Historismus und Drama}

147 Hans Hopfen: Die Meininger in Berlin. 1876 . . . . . . . . . . . 479

148 Albert Lindner: Die Meininger und ihr Kunstprincip. 1878 . . . . . . . 480

Die Gründerzeit

\section{Historismus und Nationalismus}

Kriegsmanifeste

149 Friedrich Pecht: Der Krieg und die deutsche Kunst. 1870 . . . . . . . 484

150 Berthold Auerbach: Wieder unser. 1871 . . . . . . . . . . . . . 487

Der bistorische Roman in der Nationalpädagogik

151 Wilhelm Jensen: Wilibald Alexis und die spreußische Dichtung unserer Zeit. 1866 . . . . . . . . . . . . . . . . . . . . . . . 489

152 Franz Hirsch: Geschichte und nationale Erziehung. 1882 . . . . . . . 491 
Der archaisierende Roman

153 Berthold Auerbach: Bemerkungen zu Gustav Freytag, Ingo und Ingraban. 1873493

154 Paul Lindau: Rez. Gustav Freytag, Ingo und Ingraban. 1872 • . . . . . 496

\section{Das germanische Nationalepos}

155 Wilhelm Jordan: Epische Briefe. 1876 . . . . . . . . . . . . . 501

156 Wilhelm Jordan: Das Kunstgesetz Homers und die Rhapsodik. 1869 . . . . 502

157 Ferdinand Kürnberger: Der Rhapsode Jordan. 1870 . . . . . . . . . 504

Die triviale Versepik

158 Fritz Koegel: Frauen- und Goldschnitt-Literatur. 1884 . . . . . . . . . 507

159 E. Last Literatur-Institut. Nachtrags-Verzeichniß. 1890 . . . . . . . . 509

160 Emil Kuh: Rez. Robert Hamerling, Der König von Sion. 1869 . . . . . . 510

161 Julius Hart: Julius Wolff und die ‘moderne Minnepoesie. 1887 . . . . . . . 514

\section{Weltanschauliche Positionen}

Der Spätidealismus und das Reich

162 Moriz Carriere: Die sittliche Weltordnung in den Zeichen und Aufgaben unserer Zeit. 1870 . . . . . . . . . . . . . . . . . . . . . 517

163 Moriz Carriere: Pessimismus und Idealismus in der Kunst. 1874 . . . . . 518

164 Adolf Friedrich von Schack: Nächte des Orients. 1874 . . . . . . . . . 521

165 Gustav Wacht: Emile Zola und die Commune in der Literatur. 1880 . . . 525

\section{Kritiker des Spätidealismus}

166 Leopold von Sacher-Masoch: Die Ideale unserer Zeit. 1875 . . . . . . 527

167 Ludwig Anzengruber: Dorfgänge. Eine Plauderei als Vorrede. 1879 . . . . 529

Der Pessimismus

168 Leopold von Sacher-Masoch: Das Vermächtniß Kains. Gesamtkonzept im Brief an den Bruder Karl. 1869 . . . . . . . . . . . . . . . . . . 531

169 Leopold von Sacher-Masoch: Das Vermächtniß Kains. Prolog des Wanderers. 1870 . . . . . . . . . . . . . . . . . . . . . . . 532

170 Johannes Volkelt: Rez. Siegfried Lipiner, Der entfesselte Prometheus. 1877 . 535

171 Wilhelm Jensen: Wilhelm Raabe. 1879/80 . . . . . . . . . . . . . 540

172 Eduard von Hartmann: Aphorismen über das Drama. 1870 . . . . . . . 543

173 Hieronymus Lorm: Contemplative Lyrik. 1874 . . . . . . . . . . . 545

Der germanische Tragismus

174 Felix Dahn: Odhin's Trost. 1880 . . . . . . . . . . . . . . 549 
Der Darwinismus in der Literaturtheorie

175 Carl du Prel: Psychologie der Lyrik. 1880 . . . . . . . . . . . 551

\section{Aristokratismus \\ (Heroentum, Kultur und Klassenherrschaft)}

176 Herman Grimm: Leben Michelangelo's. 1860 . . . . . . . . . . . . 554

177 Herman Grimm: Goethe. 1877 . . . . . . . . . . . . . . . . 555

178 Friedrich Nietzsche: Fünf Vorreden zu fünf ungeschriebenen Büchern. Der griechische Staat. 1872 . . . . . . . . . . . . . . . . . . . . 558

179 Heinrich von Treitschke: Der Sozialismus und seine Gönner. 1874 . . . . . 561

180 Eduard von Hartmann: Phänomenologie des sittlichen Bewußtseins. 1879 . . 565

\section{Wagner und sein Kreis \\ (Religionsstiftung, Lebensreform und Kulturkritik)}

181 Richard Wagner: Religion und Kunst. 1880 . . . . . . . . . . . . 568

182 Richard Wagner: 'Was nützt diese Erkenntniß? 1880 . . . . . . . . . 574

183 Hans von Wolzogen: Von der Sperlingsgasse bis zum Krähenfelde. 1881 . . . 577

184 Otto Glagau (?): Die Juden im Roman. 1882 . . . . . . . . . . . . 580

\section{Literarisches Leben}

\section{Schriftsteller und Gesellschaft}

Aspekte der Emanzipation des Schriftstellers

$$
\text { Auflösung der Ständegesellschaft }
$$

185 Hermann Hauff: Gedanken über die moderne schöne Literatur. 1840 . . . 586

186 Wilhelm Heinrich Riehl: Die bürgerliche Gesellschaft. Kap. Die Proletarier der

Geistesarbeit. 1851 . . . . . . . . . . . . . . . . . . . 593

Revolutionäre Organisationsentwürfe

187 Wolfgang Müller (von Königswinter): Vergangenheit und Zukunft der Kunst. 1848 .

Mäzenatentum oder Selbsthilfe

188 Franz Ferdinand Dingelstedt: Die deutsche Schillerstiftung. 1865 . . . . . 601

189 Gustav Freytag: Fürst und Künstler. 1866 . . . . . . . . . . . . . 608 
Die Entfremdung zwischen Autor und Publikum

190 Robert Prutz: Literatur und Literaturgeschichte in ihren Beziehungen zur Gegenwart. 1858 . . . . . . . . . . . . . . . . . . . . . 609

191 Friedrich Spielhagen: Produktion, Kritik und Publikum. 1883 . . . . . . 613

\section{Literatur als Ware in der Gründerzeit}

192 Wilhelm Jordan: Briefwechsel zwischen der Frau Commerzienräthin S. in Berlin und dem Dichter W. Jordan in Frankfurt. 1868 . . . . . . . . . . . 618

193 Manuscripten-Vermittelung. In: Der literarische Verkehr, 1872 . . . . . 620

194 Oswald Zimmermann: Brief an den Herausgeber der "Deutschen Dichterhalle«. 1880 . . . . . . . . . . . . . . . . . . . . . . 623

195 Correspondenz mit den Einsendern und Mitarbeitern des "Deutschen Dichterheims«. 1882 . . . . . . . . . . . . . . . . . . 624

\section{Zur Distribution der Belletristik}

Buchbesitz und nationale Gesinnung

196 Gustav Freytag: Luxus und Schönheit im modernen Leben. Die Anlage von Hausbibliotheken. 1852 . . . . . . . . . . . . . . . . . . . 626

197 Adolf Zeising: Deutsche Originalromane. 1854 . . . . . . . . . . . 630

\section{Leibbibliotheken und Romankonsum}

198 Verfügung des Königl. Preuss. Ministeriums des Innern über die Beaufsichtigung der Leihbibliotheken. 1842 . . . . . . . . . . . . . . . 633

199 Albert Last: Ueber Romane und Romanverleger. Winke für den Verleger. 1864635

200 Otto Janke: Entgegnung auf und Berichtigung $\mathrm{zu}$ "Winke für den Verleger«. 1864 . . . . . . . . . . . . . . . . . . . . . . . . . 639

201 Albert Last: Der Einfluß der Leihbibliotheken auf den Roman-Absatz. 1884 . 641

Das Klassikerjabr 1867

\section{Der Buchhandel}

202 Christian W. Wurst: Kreuz- und Querzüge in Sachen der deutschen Classiker. 1868 . . . . . . . . . . . . . . . . . . . . . . . . 647

203 Otto Mühlbrecht: Der Feldzug der deutschen Verleger im Jahre 1867.1867 • 649

Die Diskussion um den Klassikerbegriff

204 Literatur und Kunst für das Bürgerhaus. In: Die Gartenlaube, 1867 . 651

205 Rudolph Gottschall: Die Classiker als Nationaleigenthum. 1867 . . . . 654 
206 Christian W. Wurst: Kreuz- und Querzüge in Sachen der deutschen Classiker. 1868 .

207 Karl Frenzel: Die Classiker frei! 1867 . . . . . . . . . . . . . . 657

Der Kolportageroman der Gründerzeit

208 Otto Glagau: Der Colportage-Roman, oder »Gift und Dolch, Verrath und Rache ${ }_{0} 1870$. . . . . . . . . . . . . . . . . . . . . 661

Illustration und Prachtausgaben

209 Die Illustration als Hebel der Volksbildung. In: Illustrirte Zeitung, 1868 • 669

210 Friedrich Theodor Vischer: Rez. Schillers Gedichte. Jubiläumsprachtausgabe. 1861 . . . . . . . . . . . . . . . . . . . . . . . . . 671

\section{Volk und literarische Bildung}

211 Karl Gutzkow: Volk und Publicum. 1855 . . . . . . . . . . . . 674

212 Friedrich Schaubach: Zur Charakteristik der heutigen Volksliteratur. 1863 . 677

213 Moritz Müller: Göthe im Arbeiterbildungs-Verein. 1863 . . . . . . . . 679

214 Pfarrer Grob: Referat über die von der Jahresdirektion der schweiz. gemeinnützigen Gesellschaft ausgeschriebene Frage betreffend Volksliteratur. 1867 . 680 